\title{
POLIPARASSITOSI IN BAMBINO IMMIGRATO CON DIARREA ACUTA: UN CASO SINGOLARE
}

\author{
Sisino L., Fabbrizi V., Seca P.
}

Laboratorio Analisi, Sezione Microbiologia,

Ospedale Civile "Giuseppe Mazzini",

Piazza Italia, 64100 Teramo

Introduzione. Le parassitosi intestinali restano ancora grosso problema di sanità pubblica, non solo per i paesi in via di sviluppo dove sono endemiche, ma anche nei paesi industrializzati, dove la loro incidenza è in aumento per viaggi, migrazioni, adozioni.

Scopo del nostro studio è segnalare il caso singolare di un bambino di 11 anni, proveniente dall'Etiopia che, in condizioni fisiche compromesse, viene ricoverato nel Gennaio 2005 nel Reparto di Malattie Infettive per disturbi intestinali con episodi di diarrea acuta.

Metodi. Dopo i primi accertamenti clinici che rivelano ritardo nella crescita, aumento degli indici di flogosi e di anemia e quelli strumentali, quali ECO e TAC addome, che segnalano la presenza di molteplici linfoadenopatie epatiche, vengono richiesti esami microbiologici e parassitologici.

Contrariamente alla coprocoltura, risultata negativa negativa, l'esame coproparassitologico, condotto con osservazioni microscopiche dirette e dopo concentrazione, rivela la presenza di Giardia duodenalis (trofozoiti e cisti), forme vacuolate di Blastocystis hominis, rare uova di Enterobius vermicularis e cisti amebiche che, per la morfologia, le dimensioni e la presenza di non più di quattro nuclei, ci orientano verso Entamoeba histolytica/dispar.

Il bambino viene dimesso con terapia antiparassitaria, ma ad Aprile 2005, viene nuovamente ricoverato per la presenza di una tumefazione laterocervicale sx che, dopo svuotamento chirurgico, risulta essere un ascesso; sul contenuto viene richiesto l'esame colturale per germi comuni e lieviti (negativo) e per il Mycobacterium tuberculosis che risulta positivo: la colorazione di Ziehl-Neelsen rivela la presenza di BAAR e la biologia molecolare è positiva per M. tuberculosis complex; durante la degenza inizia la terapia antitubercolare che continua a domicilio.

Risultati. Le condizioni fisiche del bambino, sottoposto a controlli periodici, risultano notevolmente migliorate.

Conclusioni. Ci sembra importante segnalare questo caso di parassitosi intestinale di importazione per il crescente interesse che la problematica riveste sul nostro territorio. 